\title{
Improving X-ray Optics via Differential deposition
}

\author{
Kiranmayee Kilaru*a, Brian D. Ramsey ${ }^{\mathrm{b}}$, Carolyn Atkins ${ }^{\mathrm{c}}$ \\ anniversities Space Research Association, 320 Sparkman Dr, Huntsville, Al, USA, 35805 \\ bNASA Marshall Space Flight Center, Huntsville, Al, USA, 35805 \\ 'UK Astronomy Technology Centre, Royal Observatory, Edinburgh, EH9 3HJ, UK
}

\begin{abstract}
Differential deposition, a post-fabrication figure correction technique, has the potential to significantly improve the imaging quality of grazing-incidence X-ray optics. DC magnetron sputtering is used to selectively coat the mirror in order to minimize the figure deviations. Custom vacuum chambers have been developed at NASA MSFC that will enable the implementation of the deposition on X-ray optics. A factor of two improvement has been achieved in the angular resolution of the full-shell X-ray optics with first stage correction of differential deposition. Current efforts are focused on achieving higher improvements through efficient implementation of differential deposition.
\end{abstract}

Keywords: Differential deposition, Grazing-incidence X-ray optics

\section{INTRODUCTION}

Precision figuring of grazing-incidence X-ray optics separates naturally into initial figuring, as part of the fabrication and mounting process, and post-fabrication figure corrections. It is likely that any future fine angular resolution $\mathrm{X}$-ray mission will require both precision fabrication and figure corrections: Figure corrections aim to turn good mirrors into excellent mirrors ${ }^{1}$.

The Lynx (formerly X-ray Surveyor) mission concept, currently under study for submission to the 2020 Astrophysics Decadal Survey, is expected to conclude that Chandra-like sub-arc second angular resolution is required to meet future science goals ${ }^{2}$. This is consistent with 2013's “Enduring Quests, Daring Visions" astrophysics roadmap ${ }^{3}$ and the 2016 PCOS Technology Report ${ }^{4}$ that specifies high-resolution lightweight X-ray optics as a key enabling technology critical to progress on the highest-priority strategic astrophysics missions.

Lightweight, sub-arcsecond, X-ray optics will require substantial technological improvements over today's state of the art. For example, current production full-shell X-ray optics fabricated at Marshall Space Flight Center (MSFC) consistently achieve (X-ray-measured) $\sim 15$ arcsecond or better half-power diameter (HPD) initial performance for individual mirror shells using the nickel electroforming replication method ${ }^{5}$. Hundreds of such optics have been produced at MSFC for the HEROES hard X-ray balloon ${ }^{6}$ program (25 arcsecond HPD; 20-75 keV), FOXSI sounding rocket ${ }^{7,8}$ solar imager ( 8 arcsecond FWHM; 5-15 keV), the ART on Spectrum-Rontgen-Gamma medium energy X-ray satellite ${ }^{9}(25$ arcsecond HPD; 5-25 keV), and will be produced for the IXPE Small Explorer mission ${ }^{10}$ (25 arcsecond HPD; 2-8 keV). Even with anticipated improvements, residual stress in the fabrication process may limit the angular resolution of the best thin replicated optics to no better than $\sim 5-10$ " HPD requiring post-fabrication figure correction to achieve sub-arc second performance ${ }^{1}$.

Differential deposition, a post fabrication non-contact figure correction process pioneered by the need for microradian performance in synchrotron light facilities ${ }^{11}$, has achieved greater than a factor-of-two improvement in angular resolution. Differential deposition corrects the surface figure of a mirror by depositing material to preferentially fill in selectively coat - low regions of surface. Differential deposition is most effective at correcting the low-to mid-spatial frequency errors that are the dominant figure errors encountered in state-of-the-art full-shell replicated optics. Coating thicknesses typically range from 10's to 100's of nm.

*Kiranmayee.kilaru-1@nasa.gov 


\section{DIFFERENTIAL DEPOSITION}

Differential deposition is a technique to correct the figure errors of grazing incidence $\mathrm{x}$-ray optics. These figure errors, imparted during the fabrication of mirrors, limit the achievable angular resolution of the final mirror. Differential deposition reduces the errors by selectively depositing material on the surface of the mirror as shown schematically in Figure 1.

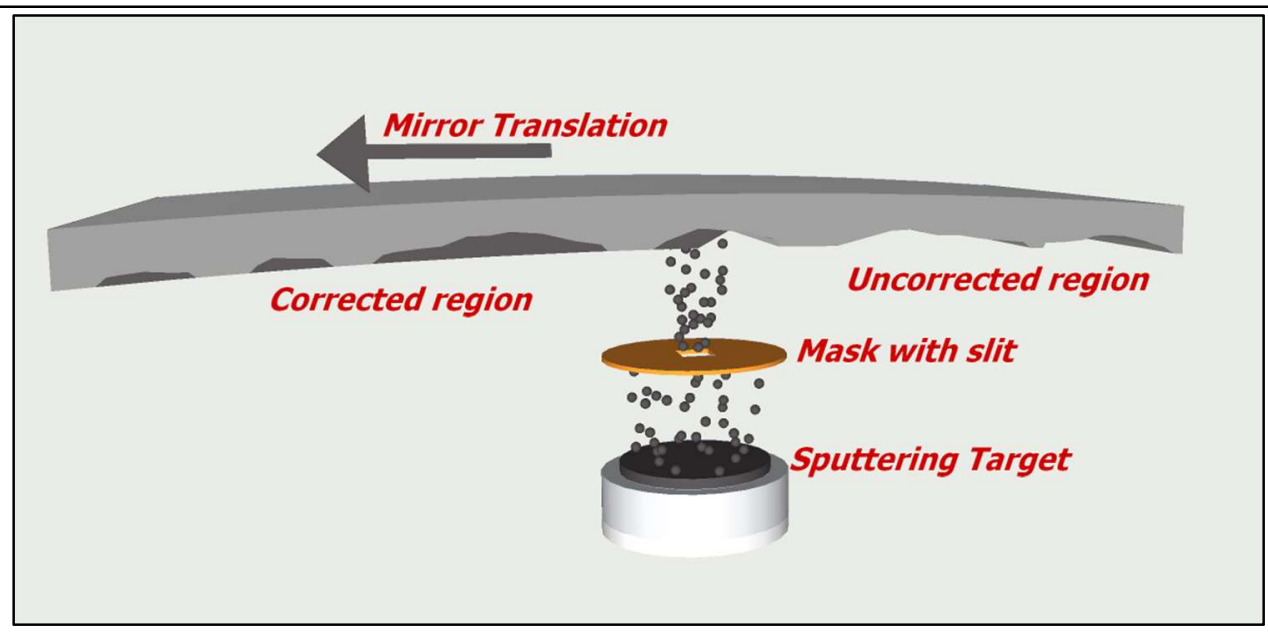

Figure 1: Schematic view of the differential-deposition correction process. A cut-away portion of an optic with exaggerated figure errors is shown that translates under computer control over the sputtering target/mask assembly (shown without confining sidewalls for clarity). Filler material is selectively deposited by controlling the translational dwell time as a function of relative mirror location.

The technique is applied in a series of steps. Briefly, these are: (1) Fabricate an X-ray optic including machining and polishing a mirror mandrel, plating (electroforming) substrate material onto the mandrel, and finally releasing the shell from the mandrel. (2) Measure surface figure and compare to desired figure thereby creating a figure error map. (3) Generate a correction map that describes the amount and location of material to be deposited. (4) Determine the optimal mask needed to define and limit the extent of the deposition to the appropriate spatial scale (a function of both the sputtering target-to-mask and mask-to-optic distances and the mask geometry; see Figure 1). (5) Apply filler material via vacuum deposition using direct current magnetron sputtering. Application of filler is through computer-controlled regulation of the speed of translation of the optic over the mask which is itself a function of the sputtering rate, desired filler thickness, and target-mask-optic-geometry.

The original figure errors for full-shell optics are typically mid-spatial frequency (few $\mathrm{mm}$ to $20 \mathrm{~mm}$ ) arising from the polishing process during mandrel fabrication and low-spatial frequency $(>20 \mathrm{~mm})$ features due to the stresses induced in the replication process; most notably in releasing the optic from the mandrel. Figure 2a shows the normalized sputtered beam profile (based on measurements) for a $5 \mathrm{~mm}$ slit size with a $10 \mathrm{~mm}$ distance between the slit and substrate. This profile is used to simulate the series of discrete steps needed to produce the desired figure correction (Figure 2b). The simulation results in a dwell sequence (Figure 2c) and a predicted deposition profile (Figure 2d). This particular example spans a $\sim 70 \mathrm{~mm}$ portion of a $600 \mathrm{~mm}$ optic, contains about 140 steps of $0.5 \mathrm{~mm} / \mathrm{step}$ on average with a range of 0.1 to 1 $\mathrm{min} / \mathrm{step}$ dwell requiring $\sim 1$ to 2 hours for the desired coating profile to be applied. Larger slits, with high deposition rates, are used to correct large-amplitude low-frequency errors. A smaller slit could be used but the process time increases accordingly. Ideally, therefore, corrections should be made in an iterative measurement/correction series using progressively smaller slits to correct progressively higher spatial frequency errors. 


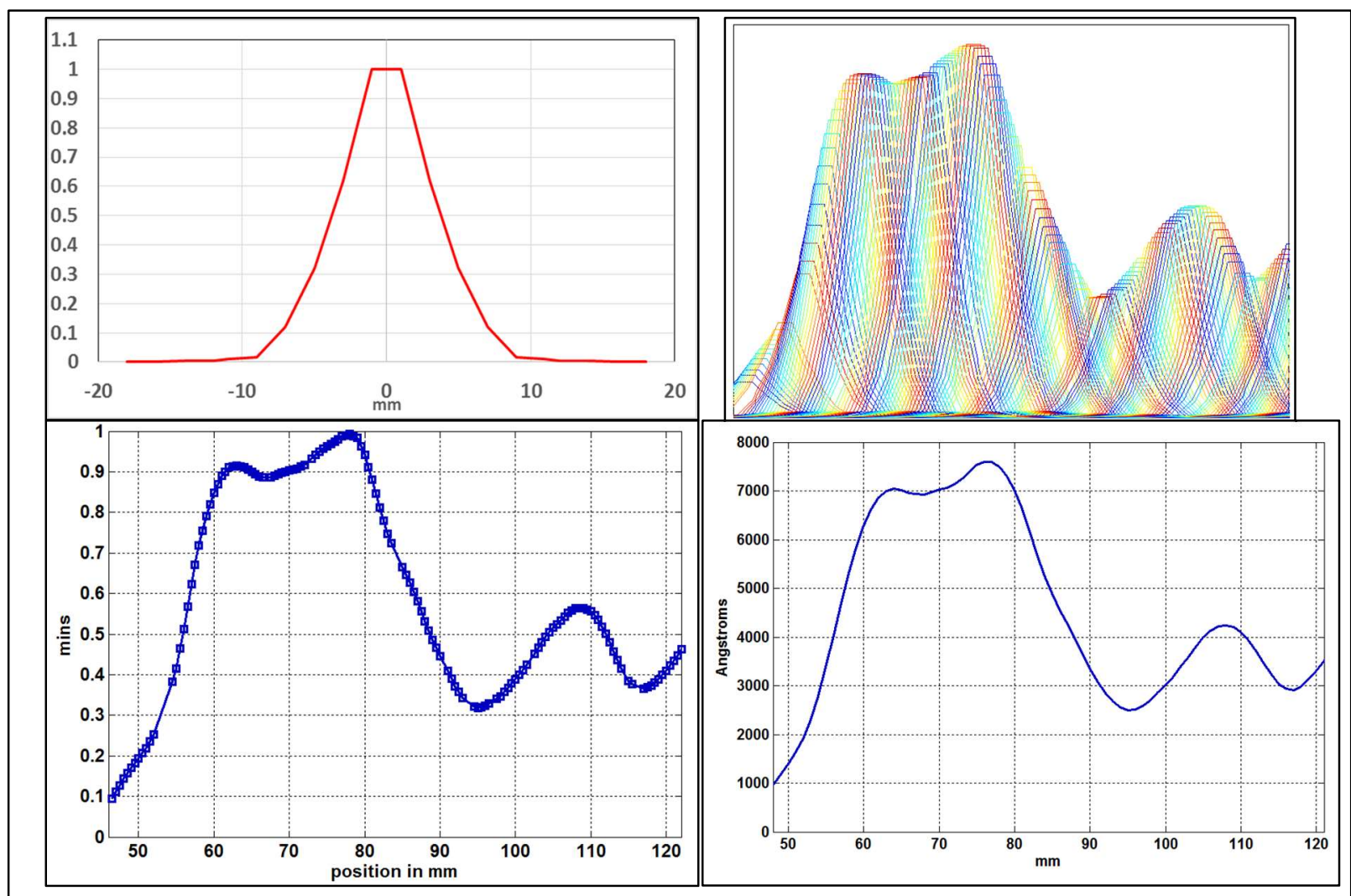

Figure 2: a) Characterized sputtered beam profile for $5 \mathrm{~mm}$ slit; b) Stepping the sputtered beam profile along the region of correction; c) Dwell time vs. position d) Simulated resultant deposition.

\section{RESULTS}

Accomplishments to date include: (1) Fully characterizing the coating chambers to determine the optimal coating parameters that provide high-quality deposits. These parameters include filler material (which depends on the substrate material and must have high deposition rate, high adhesion, and low internal stress while maintaining as-fabricated microroughness), sputtering gas pressure and sputtering power (as a result of this study, Nickel filler material with Argon sputtering gas was chosen). (2) Optimizing the mask/slit sizes and geometries to enable the desired correction profile. (3) Demonstrating that the desired and obtained profiles are in very good agreement and that the coating profile and rate of deposition have been accurately determined and that accurate corrective coatings can be applied. This demonstration included metrology and full X-ray testing of mirror performance.

\subsection{Results using profilometer metrology characterization}

Once an optic has been corrected using the differential deposition technique, it must be measured to determine the change in performance. Ideally, one shines a collimated X-beam onto the grazing incidence optic and measures its focusing properties directly. A less direct yet serviceable approach is to measure the optic's slope error (surface figure) with a profilometer and then infer the X-ray performance through ray-trace simulations. Here, we discuss progress made $\mathrm{e}^{12,13,14,15}$ using metrology and reserve discussion of direct X-ray testing to the next subsection.

Initial tests were made to correct a single meridian (a fraction of the full azimuth) of a full-shell x-ray optic, in two iterations. The first iteration used a $5 \mathrm{~mm}$ slit which corrects features of few 10's of mm spatial wavelength. A $1 \mathrm{~mm}$ slit is used for the second iteration of correction that targeted spatial wavelengths of few $\mathrm{mm}$. Figure 3 gives one such example of a measured profile that was corrected in two iterations which improved the predicted HPD, calculated from the metrology data, from 7.1 arcsecond to 2.9 arcsecond for the entire profile and from 6.7 to 1.9 arcsecond after removing 
a polynomial term representing the low-frequency error which was not corrected for. The improvement corresponds to a reduction in the calculated RMS height variation from $36 \mathrm{~nm}$ to $6.4 \mathrm{~nm}$.

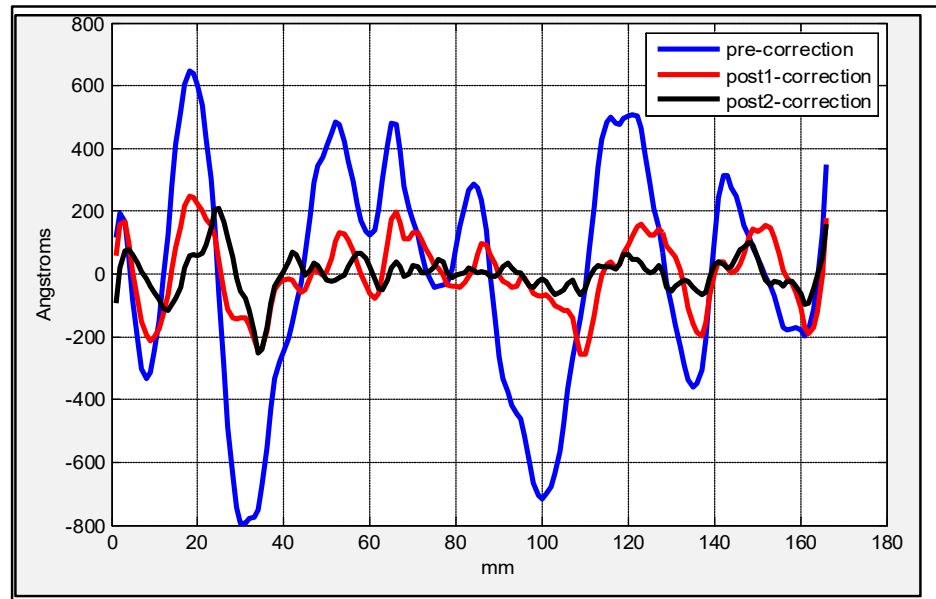

Figure 3: Mid-spatial frequency deviations in an X-ray mirror - pre and post two iterations of correction.

\subsection{Results using X-ray measurements}

The MSFC 'stray-light' facility provides a nearly parallel beam for X-ray testing shells corrected using the differential deposition technique. This facility consists of a 100-m-long vacuum tube, to which an $\mathrm{x}$-ray source is affixed on one end and the test optic and an X-ray detector are placed on the other end within a clean room facility. Figure 4 shows an intra-focus ( $40 \mathrm{~mm}$ inside the 2.7 $\mathrm{m}$ focal length) image of an X-ray shell pre- and post- correction. The length of the shell is $580 \mathrm{~mm}$ and the diameter of the shell at the midpoint is 139.6 $\mathrm{mm}$.

Three differential deposition tests were performed on this optic. Each test treats a region comprising the full length of the optic and roughly $1 / 5$ its circumference. For each test, three meridians, separated by 24 degrees, were measured to generate a correction map and each correction was applied independently (one map for each meridian). A $5 \mathrm{~mm}$ width slit was used for the differential deposition in all cases (which targets spatial features of a few 10's mm). The first test applied to meridians at $0^{\circ}, 24^{\circ}$ and $48^{\circ}$ (measured clock-wise from the top in
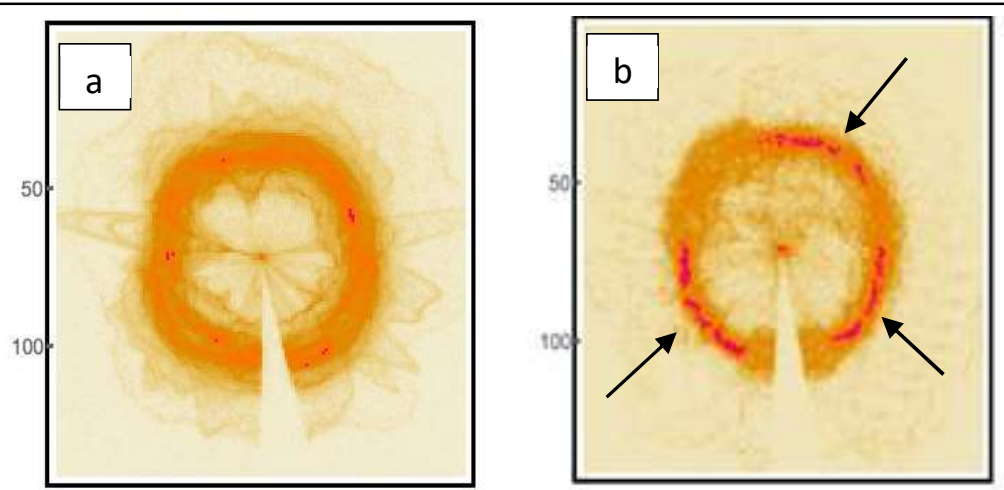

Figure 4: CCD intra-focus x-ray image pre (a) and post (b) correction. Defocusing allows one to observe the surface errors directly as a function of azimuth. Arrows on the right image point to regions corrected by differential deposition. Note the smaller annular width and more concentrated surface brightness in these regions. An occulting mask is used to block direct (unfocused) illumination. The shadow of the occulting mask support structure appears as the white triangular region in both images.

Figure 4) and used a slit length that generates a roughly $24^{\circ}$-wide azimuthal profile with a nearly uniform central $18^{0}$ portion. This test reduced the optic HPD in this region from 15.7 to 10.7 arc second. The pre- and post- error maps of the $\mathrm{P}$ and $\mathrm{H}$ segments of one of the meridians is shown in Figure 5. A similar test was applied to meridians at $216^{\circ}, 240^{\circ}$ and $264^{\circ}$. This results in improvement from 17.7 to 7.2 arcsecond HPD; a factor of $\sim 2.5$ improvement. The third test was applied to meridians at $96^{\circ}, 120^{\circ}$ and $144^{\circ}$ and was aimed at correcting only the mid-spatial frequency errors. The corrections reduced the HPD from 16.3 to 8.5 arc second. 
It is clear that an improvement of a factor of $>2$ is achieved with a single correction iteration using our differential deposition technique. As noted in the previous section, our metrology indicates additional improvements can be achieved through iterative corrections by employing a measurement-correction feedback cycle.

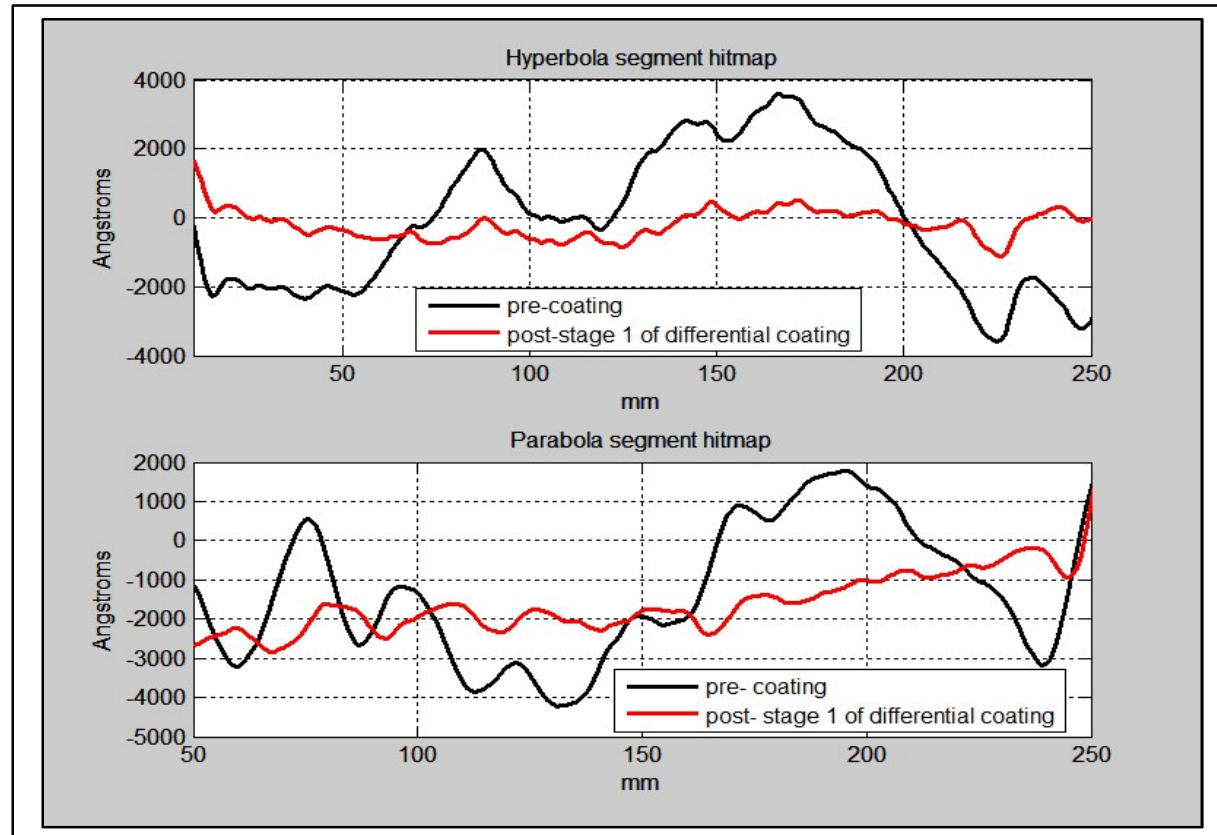

Figure 5: Error map of $\mathrm{H}$ and $\mathrm{P}$ segments pre and post correction.

\subsection{Other results}

(1) Vertical Translation System: In order to maintain a constant distance between the target and substrate for tapered mirrors, we have recently installed a vertical translation stage on which the target will be mounted - currently the mirror is held in a tilted position to keep the substrate-to-slit distance constant but this requires breaking the vacuum for coating a different meridian in order to reposition the mirror. This vertical stage will improve the efficiency by providing the ability to vary the mask to mirror distance while coating, which will enable multiple meridian correction without breaking the vacuum.

(2) Coating Induced Stress: The results obtained to date show no evidence of stress affecting or limiting the improvement achievable with differential deposition for the case of full-shell mirrors. As such, stress effect of coatings is significantly less in full shell mirrors when compared to segmented optics. An FEA analysis showed that the stress value imparted by a typical corrective coating thickness will not affect a 1 arcsecond corrected full-shell optic. In parallel, a novel in-situ stress measurement device has been developed at MSFC using which experiments are currently underway to examine methods of achieving zero intrinsic stress in thin films ${ }^{16}$.

(3) In-situ Metrology: Attempts were made to perform in-situ metrology using commercially available displacement sensors ${ }^{17}$ that have been extensively used at MSFC for stress analysis. These probes were unable to achieve the required $\sim 5 \mathrm{~nm}$ accuracy for our purposes. Thus, all the coating tests reported to date required breaking vacuum and removing the test mirror shell following differential deposition in order to perform metrology by other means before another correction iteration was possible.

\subsection{Segmented Optics}

Figure 6 shows a result of differential deposition along the axial profile of a segmented optics - a slumped glass segment fabricated at NASAGSFC. The measured profile after coating is shown in the lower plot of Figure 6 . The after- 
coating and the simulation show good fidelity across the length of the optic. The rms height before and after correction is $200 \AA$ and $96 \AA$ respectively and demonstrating a factor of 2 improvement in height rms from a single correction.

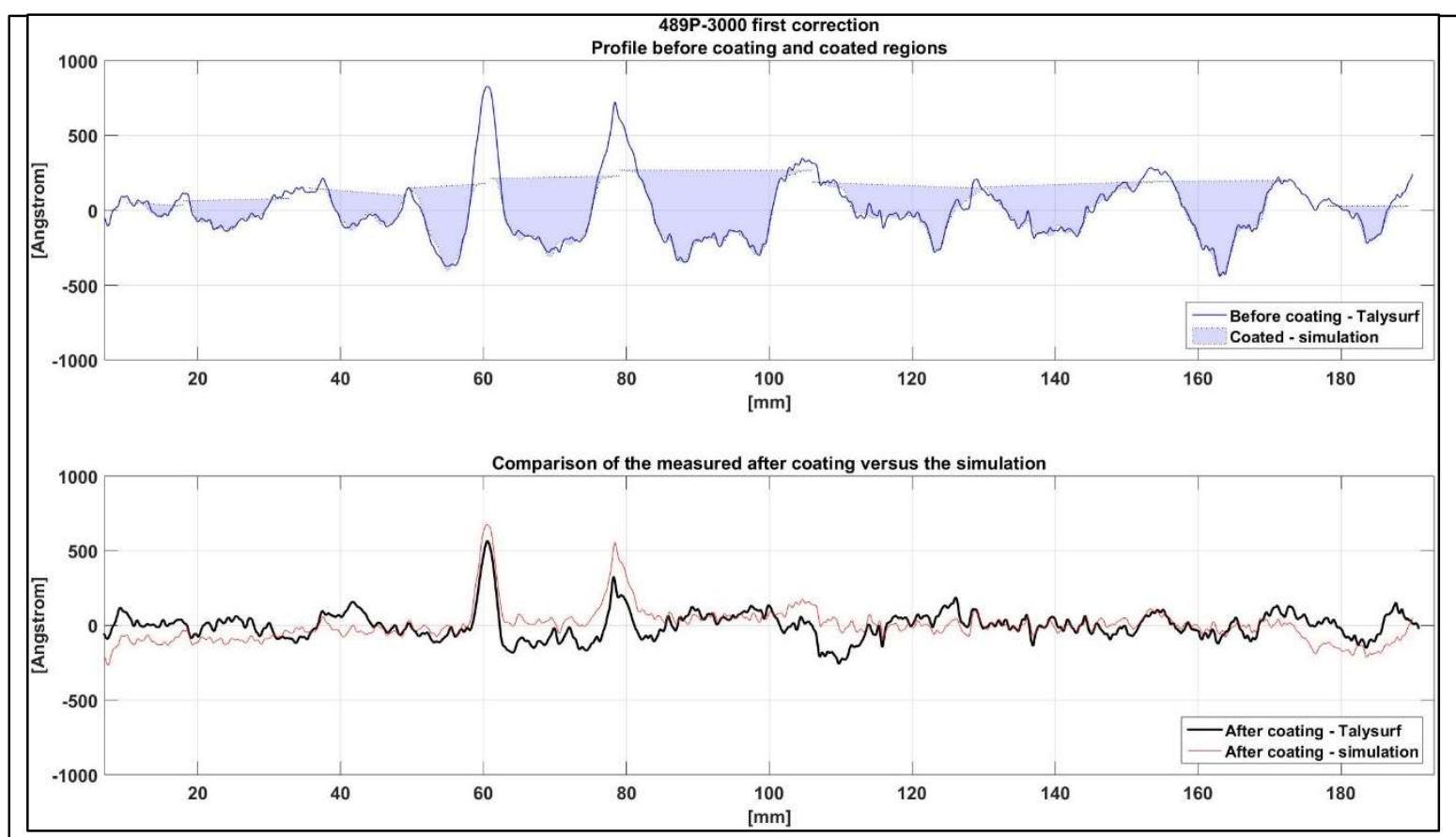

Figure 6: Differential deposition on segmented optics: The upper plot depicts the before correction where the shaded regions highlight where differential deposition has been applied. The lower plot compares the after correction measurement with the after correction simulation.

\section{FUTURE WORK}

We have demonstrated greater than a factor-of-two improvement in the X-ray performance of full-shell X-ray optics using our selective coating differential deposition technique applied to low- and mid- frequency figure deviations. Or future efforts are focused on achieving further improvement by the efficient implementation of additional correction cycles that address successively higher spatial frequency figure errors. Table 1 below describes the improvement in optical performance expected from 4-iteration measurement-correction implementation. Beginning with a typical 15 arcsecond optic, a 1 arc second optic is theoretically feasible using the differential deposition process. However, such large improvements have yet to be achieved in practice.

Table 1. Improvement in optical performance expected from 4-iteration measurement-correction implementation.

\begin{tabular}{|c|c|c|c|c|}
\hline Cycle & $\begin{array}{c}\text { Amplitude of } \\
\text { Correction }\end{array}$ & $\begin{array}{c}\text { Spatial-Frequency of } \\
\text { Correction }\end{array}$ & Average Slit-Size & $\begin{array}{c}\text { Predicted Performance } \\
\text { Improvement in } \\
\text { arcsecond }\end{array}$ \\
\hline 1 & $>1000 \AA$ & 50 to 100 's $\mathrm{mm}$ & $10 \mathrm{~mm}$ & From 15 to 7 \\
\hline 2 & $=/<1000 \AA$ & 20 to $50 \mathrm{~mm}$ & $5 \mathrm{~mm}$ & From 7 to 3 \\
\hline 3 & 100 's of $\AA$ & 10 to $20 \mathrm{~mm}$ & $1 \mathrm{~mm}$ & From 3 to 1.5 \\
\hline 4 & $=/<100 \AA$ & $<10 \mathrm{~mm}$ & $0.5 \mathrm{~mm}$ & From 1.5 to $<1$ \\
\hline
\end{tabular}

Currently, after the first correction iteration, (1) the optic must be removed from the vacuum chamber to measure its post-correction surface figure and (2) the chamber must be opened to modify the slit-configuration. This makes multiple cycles of correction a time consuming process and can introduce alignment errors. These errors will propagate to 
subsequent correction iterations. We are investigating a more efficient and a more accurate approach for multiple correction iterations that addresses both these issues: (1) In-situ metrology and (2) active-slit adjustment.

a) Slope measuring in-situ metrology: We are considering to implement an in-situ metrology approach similar to the proven and routinely-utilized metrology procedure at MSFC, called vertical long trace profiler (VLTP) ${ }^{18}$, which is a slope-measuring instrument widely used for measuring surface figure and slope errors on grazing incidence X-ray mirrors used in synchrotron facilities and X-ray astronomy. A schematic of the metrology system is shown in Figure 7 . The optical board consists of a non-polarizing beam splitter and porro prisms, which split the incoming beam into 2 collinear beams which again pass through a polarizing- beam splitter that splits the beam-pair into two paths. One path is directed to a reference mirror and the other to the surface under test. Light from both these paths is reflected back and passes through a Fourier transform lens to focus onto a CCD detector. The two collinear beams of each path form an interference pattern at the detector, the minima of which is used to locate the beam position on the detector. To determine the relative slope between the scan points of the test surface, a change in the relative positions of the intensity pattern minima is measured (in software). The reference slope obtained from the stationary mirror is subtracted from the test surface slope to remove any pointing instability. For the in-situ set-up laser light to the test surface will pass through an optical feed-through flange into the vacuum and a pentaprism will be used to direct the light to and from the surface of the optic. The pentaprism will be held stationary inside the chamber while the optic is translated over it (in analogy to the relation between the sputtering target and the optic).

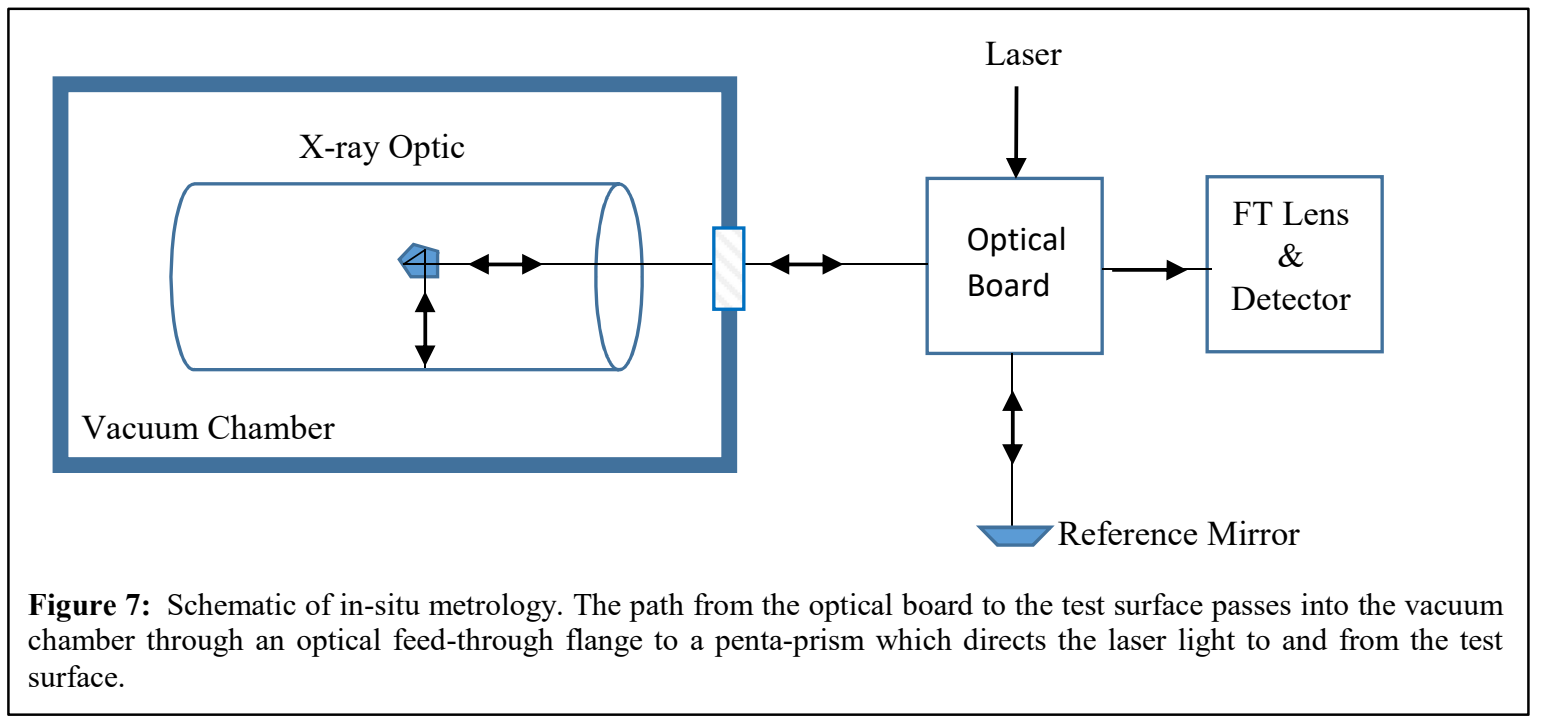

b) Active-slit control: Correcting different spatial frequencies requires different slit widths; typically, smaller widths are required for correcting higher spatial frequency errors. We are investigating the implementation of an activeslit control system, i.e., implement the capability of changing the slit width in conjunction with the (computer-controlled) translation/dwell stages. This has multiple advantages including: In combination with in-situ metrology, multiple iterations of correction can be performed without breaking vacuum; In combination with the vertical stage, the slit-optic geometry can be controlled to greater precision; The slit width can be varied to correct multiple spatial frequencies in a single iteration.

A preliminary design concept has been prepared for this approach and a 3D drawing of this is shown in Figure 8 . The shutter blades that make up the slit width are mechanically arranged such that they can be moved equally towards or away from the center. The blades are translated through vacuum compatible motors that can be computer controlled from outside the vacuum chamber. The active-slit approach in combination with the in-situ metrology will not only improve the efficiency of multiple stage corrections but also the accuracy of correction, since the mirror will be corrected in the same position as it is measured. 


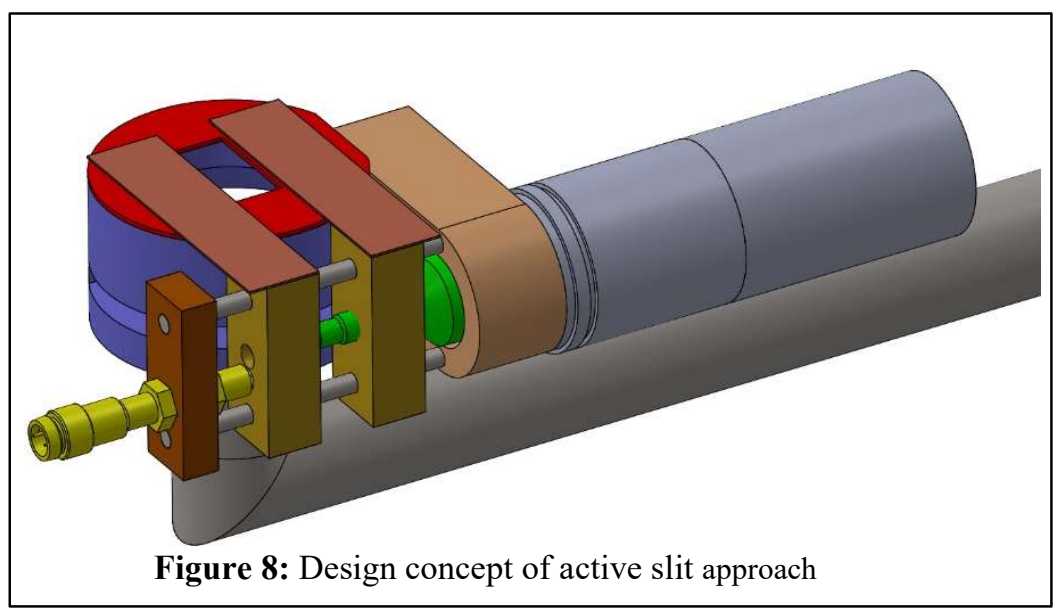

\section{CONCLUSION}

Research on the accurate and efficient implementation of differential deposition - a post fabrication figure correction technique for X-ray optics, is being actively pursued at NASA MSFC. Custom designed vacuum chambers have been installed and characterized. A factor of two improvement in the performance of full-shell optics has been demonstrated through X-ray testing. Future efforts will focus on achieving higher improvements with the efficient implementation of the technique through in-situ metrology and active-slit approach.

\section{REFERENCES}

[1] O'Dell, S. L., et al. 2014, "Toward large-area sub-arcsecond x-ray telescopes", Adaptive X-Ray Optics III 9208, 920805.

[2] Gaskin, J., Ozel, F., Vikhlinin, A. 2016, "The X-Ray Surveyor mission concept study: forging the path to NASA astrophysics 2020 decadal survey prioritization”, SPIE Conference Series 9904, 99040N.

[3]https://science.nasa.gov/science-committee/subcommittees/nac-astrophysics-subcommittee/astrophysics-roadmap

[4] http://pcos.gsfc.nasa.gov/technology/documents/PCOS2016PATR.pdf

[5] O'Dell, S. L., et al. 2015, "X-ray optics at NASA Marshall Space Flight Center", EUV and X-ray Optics: Synergy between Laboratory and Space IV 9510, 951003.

[6] Gaskin, J. A., et al. 2014, "SuperHERO: the next generation hard x-ray HEROES telescope", Space Telescopes and Instrumentation 2014: Ultraviolet to Gamma Ray 9144, 91443Z.

[7] Krucker, S., et al. 2014, "First Images from the Focusing Optics X-Ray Solar Imager”, ApJ 793, L32.

[8] Christe, S., et al. 2016, "FOXSI-2: Upgrades of the Focusing Optics X-ray Solar Imager for its Second Flight”, Journal of Astronomical Instrumentation, 5, 1640005-625.

[9] Pavlinsky, M., et al. 2016, "Status of ART-XC/SRG instrument”, SPIE Conference Series 9905, 99051J.

[10] Weisskopf, M. C., et al. 2016, "The Imaging X-ray Polarimetry Explorer (IXPE)”, SPIE Conference Series 9905, 990517.

[11] Alcock, S. G. \& Cockerton, S. 2010, "A preferential coating technique for fabricating large, high quality optics", Nuclear Instruments and Methods in Physics Research A 616, 110-114. 
[12] Kilaru, K., et al. 2015, "Progress in differential deposition for improving the figures of full-shell astronomical grazing incidence x-ray optics”, SPIE Conference Series 9603, 96031F.

[13] Kilaru, K., Ramsey, B. D., Gubarev, M. V., Gregory, D. A. 2011, "Differential deposition technique for figure corrections in grazing-incidence x-ray optics", Optical Engineering 50, 106501-106501.

[14] Kilaru, K., et al. 2011, "Differential deposition to correct surface figure deviations in astronomical grazing-incidence x-ray optics", SPIE Conference Series 8147, 81470X.

[15] Kilaru, K. 2010, "An investigation of differential deposition for figure corrections in grazing incident X-ray optics", Ph.D. Thesis

[16] Broadway, D. M., et al. 2015, “Achieving zero stress in iridium, chromium, and nickel thin films”, EUV and X-ray Optics: Synergy between Laboratory and Space IV 9510, 95100E.

[17] www.philtec.com

[18] Gubarev, M. V., Thomas Kester, T., Takacs, P. Z. 2001 "Calibration of a vertical-scan long trace profiler at MSFC", Optical Manufacturing and Testing IV, 4451, 333. 